\title{
Gene of the month: IDH1
}

\author{
Cassandra Bruce-Brand ${ }^{1,2}$ Dhirendra Govender (10) 3,4
}

${ }^{1}$ Division of Anatomical Pathology, Stellenbosch University Faculty of Medicine and Health Sciences, Cape Town, Western Cape, South Africa

${ }^{2}$ Anatomical Pathology, National Health Laboratory Service, Tygerberg Hospital, Cape Town, Western Cape, South Africa ${ }^{3}$ Anatomical Pathology, Pathcare Cape Town, Cape Town, South Africa

${ }^{4}$ Division of Anatomical Pathology, University of Cape Town, Cape Town, Western Cape, South Africa

\section{Correspondence to}

Dr Cassandra Bruce-Brand Division of Anatomical Pathology, Stellenbosch University Faculty of Medicine and Health Sciences, Cape Town 7602 , South Africa; cassandra. bruce-bran@nhls.ac.za

Accepted 7 July 2020 Published Online First 29 July 2020

\section{Check for updates}

(c) Author(s) (or their employer(s)) 2020. No commercial re-use. See rights and permissions. Published by BMJ.

To cite: Bruce-Brand C, Govender D. J Clin Pathol 2020:73:611-615.

\section{ABSTRACT}

Isocitrate dehydrogenase 1 (IDH1) encodes a protein which catalyses the oxidative decarboxylation of isocitrate to $\alpha$-ketoglutarate. Mutant IDH1 favours the production of 2-hydroxyglutarate, an oncometabolite with multiple downstream effects which promote tumourigenesis. IDH1 mutations have been described in a number of neoplasms most notably low-grade diffuse gliomas, conventional central and periosteal cartilaginous tumours and cytogenetically normal acute myeloid leukaemia. Post zygotic somatic mutations of IDH1 characterise the majority of cases of Ollier disease and Maffucci syndrome. IDH1 mutations are uncommon in epithelial neoplasia but have been described in cholangiocarcinoma.

\section{INTRODUCTION}

Isocitrate dehydrogenases (IDHs) are enzymes that catalyse the oxidative decarboxylation of isocitrate to $\alpha$-ketoglutarate. ${ }^{12}$ IDH exists in three isoforms in humans (IDH1, IDH2 and IDH3). ${ }^{3}$ IDH1 (cytosolic) and IDH2 (mitochondrial) are nicotinamide adenine dinucleotide phosphate $\left(\mathrm{NADP}^{+}\right)$dependent while IDH3 (mitochondrial) participates in the citric acid cycle and is nicotinamide adenine dinucleotide $\left(\mathrm{NAD}^{+}\right)$dependent. IDH1 and IDH2 are the isoforms implicated in tumourigenesis. Mutations in IDH2 occur in up to $40 \%$ of angioimmunoblastic T-cell lymphoma ${ }^{4}$ and occur more frequently in cytogenetically normal acute myeloid leukaemia (AML) than IDH1 mutations. ${ }^{5}$ IDH2 mutations are also implicated in a subset of chondrosarcoma, ${ }^{6}$ cholangiocarcinoma ${ }^{7}$ and low-grade diffuse gliomas. ${ }^{89}$ This review will focus on IDH1.

\section{STRUCTURE}

The IDH1 gene is located at chromosome $2 \mathrm{q} 34$ and contains 10 exons that span $18.9 \mathrm{~kb}^{10}{ }^{11}$ It encodes the IDH1 protein which comprises 414 amino acids with a molecular mass of $46.7 \mathrm{kD}$ and is located within cytoplasm and peroxisomes. ${ }^{12}$ IDH1 is a homodimer comprising two hydrophilic active sites and two protein subunits or monomers. ${ }^{13}$ Each monomer is made up of a large domain, a small domain and a clasp domain with two clefts (figure 1). ${ }^{13}$ The deep cleft which lies between the large domain and small domain of one monomer and the small domain of the second monomer forms the active $\mathrm{NADP}^{+}$binding site. ${ }^{13}$

\section{FUNCTION}

As discussed above, IDH1 catalyses the oxidative decarboxylation of isocitrate to $\alpha$-ketoglutarate (figure 2). ${ }^{12}$ This process is $\mathrm{NADP}^{+}$dependent and results in reduced nicotinamide adenine dinucleotide phosphate (NADPH). ${ }^{1}$ This reaction is reversible under physiological conditions. ${ }^{14}$ Production of cytoplasmic NADPH reduces intracellular oxidative stress while $\alpha$-ketoglutarate maintains DNA and histone proteins in a demethylated state. ${ }^{131516}$ IDH1 generates the NADPH required for cholesterol and fatty acid biosynthesis and plays a critical role in glucose-stimulated insulin secretion. ${ }^{11} 17$ Acetyl-coenzyme A synthesis from $\alpha$-ketoglutarate under hypoxic conditions is required for lipogenesis. ${ }^{18}$

\section{MUTANT IDH1}

IDH1 mutations are heterozygous oncogenic gain of function mutations resulting in one mutant and one wild-type allele. ${ }^{19}$ Recurrent missense mutations lead to a single amino-acid substitution of arginine at codon 132 in exon $4 .{ }^{19}$ The most common mutation variants include p.R132H and p.R132S although p.R132C, p.R132G and p.R132L have also been described. ${ }^{20}{ }^{21}$ Mutation decreases the binding affinity of the active sites for isocitrate and increases their affinity for NADPH thereby impairing decarboxylation of isocitrate (the 'forward' reaction). ${ }^{14}$ The 'reverse' reaction is favoured but incomplete when IDH1 is mutated resulting in the production of the R-enantiomer of 2-hydroxyglutarate (2HG), an oncometabolite (figure 2). ${ }^{14}{ }^{22}$ Furthermore, mutant IDH1 (mIDH1) production of (R)-2HG is enhanced by coexpression of wild-type IDH1 which provides the $\alpha$-ketoglutarate substrate required for the reaction. ${ }^{1423}$ Production of the R-enantiomer of $2 \mathrm{HG}$ has numerous downstream effects which promote tumourigenesis including inhibition of histone demethylation. The latter inhibits differentiation of progenitor cells and stimulates Egl nine homolog 1 which leads to diminished hypoxia-inducible factor. ${ }^{2324}$ IDH1 mutation also results in a global DNA hypermethylator signature through inhibition of the ten-eleven translocation family and the Jumonji family of histone lysine demethylases. ${ }^{23-26}$

\section{GLIOMAS}

The overwhelming majority of WHO grade II or III diffuse gliomas (diffuse astrocytoma, anaplastic astrocytoma, oligodendroglioma, anaplastic oligodendroglioma) demonstrate IDH1 or IDH2 mutations. ${ }^{892027}$ The majority of glioblastomas (WHO Grade IV) are IDH-wild type; however, those arising from low-grade gliomas (so-called secondary glioblastomas) are $I D H$ mutant. $^{927} 28$ In keeping with this, $I D H$-mutant glioblastomas occur in younger patients and demonstrate a better prognosis than wild-type tumours. ${ }^{20}$ The latest WHO classification of central nervous system tumours update of 2016 classifies glial tumours using an 


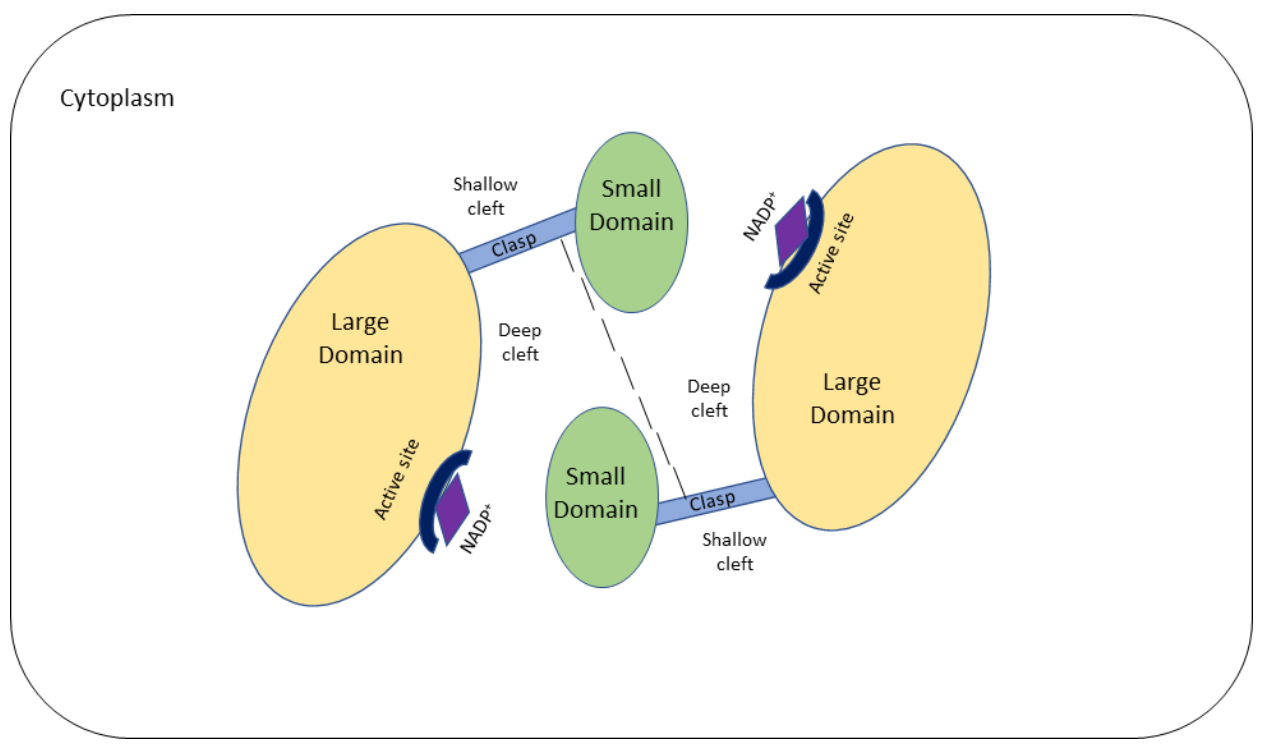

Figure 1 Simplified schematic representation of isocitrate dehydrogenase (IDH1) in its open, inactive state. The IDH1 protein comprises two monomers which each consist of a large domain, small domain and clasp domain. Two clefts are present on either side of the clasp domain: a shallow and a deep cleft. The deep cleft lies between the large and small domain of one monomer and the small domain of the second monomer. The deep cleft contains the active binding site for NADP ${ }^{+}$. The two clasp regions join the two monomers together. NADP ${ }^{+}$, nicotinamide adenine dinucleotide phosphate.

integrated genotype-phenotype approach based on the presence or absence of $I D H$ mutation (IDH mutant or IDH wild type). ${ }^{29}$ The IDH1 R132H mutation is the most common in this setting (90\% of IDH mutations) and an immunohistochemical stain against the mutant-specific antigen (R132H-mutant IDH1) has been developed (figure 3). ${ }^{30}$ This has become an essential tool in the workup of glial tumours and may also be used to distinguish a true glial neoplasm from reactive gliosis. ${ }^{31} 32$ Positive nuclear and cytoplasmic staining is seen in the majority of cells in $I D H$ mutant tumours. ${ }^{33}$ IDH1 R132H immunohistochemistry should be performed in all diffuse gliomas followed by IDH1 and IDH2 sequencing of all negative low-grade gliomas and glioblastomas in patients younger than 55 years. ${ }^{34}$

\section{CARTILAGINOUS NEOPLASMS}

Heterozygous IDH1 mutations have been demonstrated in $51 \%$ of conventional central and periosteal cartilaginous neoplasms including enchondroma, chondrosarcoma (grades 1-3), dedifferentiated chondrosarcoma, periosteal chondroma and periosteal chondrosarcoma. ${ }^{6}$ These mutations are absent in peripheral chondrosarcomas (associated with EXT1 and EXT2

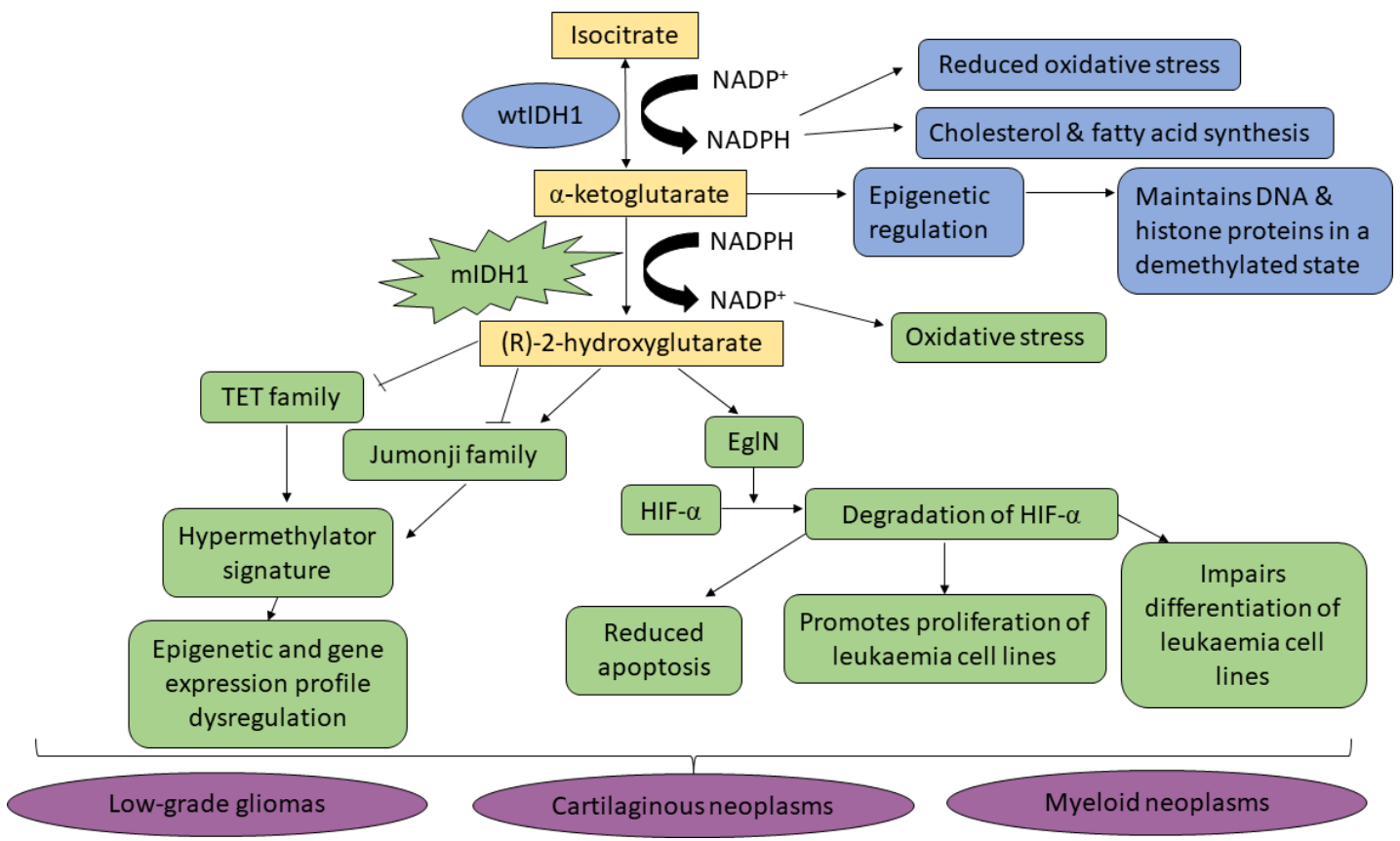

Figure 2 Schematic representation of the function of isocitrate dehydrogenase (IDH1). EgIN, Egl nine homolog 1; HIF, hypoxia-inducible factor; mIDH1, mutant IDH1; NAD, nicotinamide adenine dinucleotide; NADPH, nicotinamide adenine dinucleotide phosphate; wtIDH1, wild-type IDH1. 


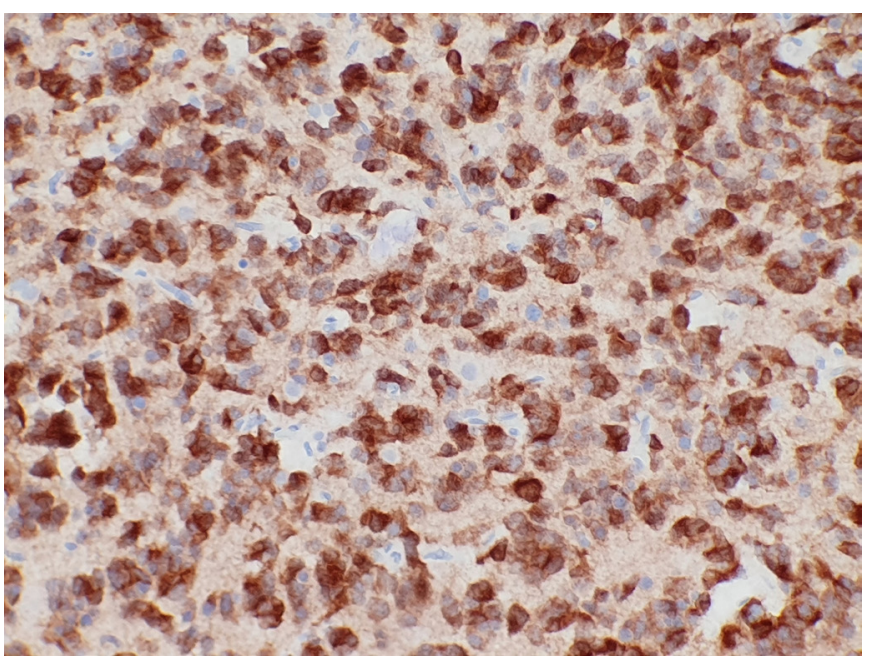

Figure 3 Isocitrate dehydrogenase (IDH1) R132H immunohistochemical stain showing cytoplasmic and nuclear staining confirming an IDH1-mutant astrocytoma.

mutations) and osteosarcoma. ${ }^{635}$ The presence of IDH1 mutation has been shown to be a useful tool to distinguish chondrosarcoma from chondroblastic osteosarcoma and dedifferentiated chondrosarcoma with osteosarcomatous differentiation from osteosarcoma. ${ }^{36}$

\section{ENCHONDROMATOSIS: OLLIER DISEASE AND MAFFUCCI SYNDROME}

Enchondromatosis is a rare heterogenous disorder characterised by the presence of multiple symptomatic intramedullary cartilaginous neoplasms. Ollier disease and Maffucci syndrome are the most common subtypes and are typically non-familial disorders. Both disorders demonstrate multiple enchondromas involving the tubular bones of the limbs with an increased risk for development of secondary chondrosarcoma. Maffucci syndrome is characterised by the addition of soft tissue, visceral or cutaneous haemangiomas particularly spindle cell haemangiomas. ${ }^{37}$

IDH1 mutations have been described in the tumours of $85 \%$ of patients with Ollier disease and $81 \%$ of those with Maffucci syndrome. ${ }^{38} 39$ These postzygotic mutations are present in the enchondromas, chondrosarcomas and spindle cell haemangiomas of the afflicted with identical mutations identified in multiple tumour types from the same patient. ${ }^{38} 39$ Mutations described include R132C, R132H and R132G in exon $4 .{ }^{38} 39$ Maffucci syndrome shows exclusively R132C mutations. A low frequency of $\mathrm{mIDH} 1$ has been described in normal tissue from these patients. ${ }^{38}$

\section{SPINDLE CELL HAEMANGIOMA}

First described as spindle cell haemangioendothelioma, spindle cell haemangioma is now classified as a benign vascular neoplasm based on its excellent prognosis. ${ }^{4041}$ When occurring outside the setting of multiple enchondromas, IDH1 mutations are seen in $64 \%$ of cases, a feature that has not been demonstrated in any other vascular lesions or malformations. ${ }^{42}$

\section{MYELOID NEOPLASMS}

$\mathrm{mIDH} 1$ has been reported in AML (7\%-14\%), ${ }^{43}$ acute lymphoblastic leukaemia $(5.5 \%),{ }^{44}$ myelodysplastic syndromes (MDS; $3 \%)^{45}$ and myeloproliferative neoplasms (MPN). Most IDH1 mutations described in this setting involve a cysteine or histidine
Table 1 Neoplasia frequently associated with IDH1 mutations

\begin{tabular}{lll}
\hline Neoplasm & Cases with IDH1 mutation (\%) & $\begin{array}{l}\text { Most frequent IDH1 } \\
\text { mutation type }\end{array}$ \\
\hline Gliomas-low grade, diffuse & $80 \%^{8920}$ & R132H \\
\hline Cytogenetically normal AML & $7 \%-14 \%^{43}$ & R132C, R132H \\
\hline Enchondromas & $52 \%$ (sporadic) & R132C, R132H \\
& $90 \%$ (enchondromatosis) $)^{63839}$ & \\
\hline Spindle cell haemangioma & $64 \%{ }^{42}$ & R132C \\
Cholangiocarcinoma & $20 \%-35 \%{ }^{751}$ & R132C, R132L, R132G, \\
& & R132S \\
\hline
\end{tabular}

AML, acute myeloid leukaemia; IDH, isocitrate dehydrogenase.

substitution for arginine at R132 (R132C or R132H). IDH2 mutations are more common than IDH1 mutations in AML and $\mathrm{MDS}^{43} \mathrm{mIDH} 1$ in AML is associated with cytogenetically normal AML, cytogenetically intermediate-risk AML and trisomy $8 .^{43}$ Approximately $20 \%$ of MPN at leukaemic transformation show IDH1/2 mutations. ${ }^{46}$

The prognostic significance of IDH1 mutations in AML has been controversial. ${ }^{47}$ A large meta-analysis showed reduced overall survival and a lower rate of complete remission with cytotoxic chemotherapy. ${ }^{48} \mathrm{mIDH} 1$ in MDS has a negative prognostic impact with reduced overall survival and higher rates of leukaemic transformation. ${ }^{49}$

\section{CHOLANGIOCARCINOMA}

Cholangiocarcinoma is a malignant tumour arising from biliary tract epithelium and can be classified as intrahepatic or extrahepatic based on anatomical location. ${ }^{50}$ The prognosis is poor with most patients demonstrating advanced disease at presentation. ${ }^{50}$ IDH1 mutations occur in $20 \%-35 \%$ of intrahepatic cholangiocarcinoma and only rarely in extrahepatic cholangiocarcinoma. ${ }^{751}$ Mutations described include R132C, R132L, R132G and R132S. ${ }^{7}$

\section{OTHER NEOPLASMS}

Apart from cholangiocarcinoma, IDH1 mutations are rare in epithelial tumours but have been reported in a small subset of prostate adenocarcinoma $(2.5 \%)^{52}$ and non-small cell lung carcinoma $(0.6 \%) .{ }^{53}$ Novel IDH1 mutations have also been described in one case each of anaplastic thyroid carcinoma (G123R) and follicular thyroid cancer (V71I). ${ }^{54}$ Two of 39 malignant melanomas in one study showed IDH1 mutations, both of which occurred with either BRAF or KIT mutations. ${ }^{55}$ See table 1 for a summary of neoplasms frequently associated with IDH1 mutations.

\section{TARGETED THERAPY}

A number of strategies for targeted therapy in IDH1 mutant tumours have been investigated including hypomethylating agents, IDH mutant enzyme inhibitors, immunotherapy and BCL-2 inhibition. Preclinical studies have validated the proof of concept that targeted inhibition of IDH1 mutants results in decreased 2-HG, release of cellular differentiation block and reversal of histone and DNA hypermethylation. ${ }^{5657}$ Four IDH1 inhibitors are currently under investigation in clinical trials for treatment of AML, gliomas and solid tumours. AG-120 (Ivosidenib, Tibsovo), an oral small-molecule inhibitor of mutant IDH1, was shown to have an acceptable safety profile when used as monotherapy for advanced solid tumours. ${ }^{58}$ AG-881 (Vorasidenib, an oral pan-mutant IDH1/2 inhibitor), BAY1436032 and 
DS-1001b (both IDH1 mutant inhibitors) are still under investigation to determine their safety profiles. ${ }^{59-61}$

\section{Take home messages}

- Wild-type isocitrate dehydrogenase (IDH1) converts isocitrate to $\alpha$-ketoglutarate. Mutant IDH1 converts $\alpha$-ketoglutarate to the R-enantiomer of 2-hydroxyglutarate, an oncometabolite that results in tumourigenesis.

- IDH1 mutations characterise low-grade diffuse glial neoplasms and are present in glioblastomas arising from lowgrade gliomas. These patients have a better prognosis than IDH-wild type glioblastoma.

- Nonfamilial postzygotic IDH1 mutations are present in the majority of patients with Ollier disease and Maffucci syndrome which results in multiple enchondromas and an increased risk of secondary chondrosarcoma.

- Cytogenetically normal acute myeloid leukaemia and some other myeloid neoplasms demonstrate IDH1 mutations.

- IDH1 mutations are also present in cholangiocarcinoma but are rare in other epithelial neoplasms.

\section{Handling editor Runjan Chetty.}

Contributors Both authors contributed equally.

Funding The authors have not declared a specific grant for this research from any funding agency in the public, commercial or not-for-profit sectors.

Competing interests None declared.

Patient consent for publication Not required.

Provenance and peer review Commissioned; internally peer reviewed.

\section{ORCID iD}

Dhirendra Govender http://orcid.org/0000-0003-1487-8255

\section{REFERENCES}

1 Geisbrecht BV, Gould SJ. The human PICD gene encodes a cytoplasmic and peroxisomal NADP(+)-dependent isocitrate dehydrogenase. J Biol Chem 1999;274:30527-33

2 Koshland DE, Walsh K, LaPorte DC. Sensitivity of metabolic fluxes to covalent control. Curr Top Cell Regul 1985;27:13-22.

3 Dalziel K. Isocitrate dehydrogenase and related oxidative decarboxylases. FEBS Lett 1980;117(Suppl):K45-55.

4 Cairns RA, Iqbal J, Lemonnier F, et al. IDH2 mutations are frequent in angioimmunoblastic T-cell lymphoma. Blood 2012;119:1901-3.

5 Marcucci G, Maharry K, Wu Y-Z, et al. IDH1 and IDH2 gene mutations identify novel molecular subsets within de novo cytogenetically normal acute myeloid leukemia: a cancer and leukemia group B study. J Clin Oncol 2010;28:2348-55.

6 Amary MF, Bacsi K, Maggiani F, et al. IDH1 and IDH2 mutations are frequent events in central chondrosarcoma and central and periosteal chondromas but not in other mesenchymal tumours. J Pathol 2011;224:334-43.

7 Kipp BR, Voss JS, Kerr SE, et al. Isocitrate dehydrogenase 1 and 2 mutations in cholangiocarcinoma. Hum Pathol 2012;43:1552-8.

8 Hartmann C, Meyer J, Balss J, et al. Type and frequency of IDH1 and IDH2 mutations are related to astrocytic and oligodendroglial differentiation and age: a study of 1,010 diffuse gliomas. Acta Neuropathol 2009;118:469-74.

9 Yan $\mathrm{H}$, Parsons DW, Jin G, et al. IDH1 and IDH2 mutations in gliomas. N Engl J Med 2009;360:765-73.

10 Creagan RP, Carritt B, Chen S, et al. Chromosome assignments of genes in man using mouse-human somatic cell hybrids: cytoplasmic isocitrate dehydrogenase (IDH 1) and malate dehydrogenase (MDH 1) to chromosomes 2. Am J Hum Genet 1974;26:604-13.

11 Shechter I, Dai P, Huo L, et al. IDH1 gene transcription is sterol regulated and activated by SREBP-1a and SREBP-2 in human hepatoma HepG 2 cells: evidence that IDH1 may regulate lipogenesis in hepatic cells. J Lipid Res 2003;44:2169-80.

12 Hamosh A. OMIM, isocitrate dehydrogenase 1. Available: https://www.omim.org/ entry/147700

$13 \mathrm{Xu} \mathrm{X}$, Zhao J, Xu Z, et al. Structures of human cytosolic NADP-dependent isocitrate dehydrogenase reveal a novel self-regulatory mechanism of activity. J Biol Chem 2004;279:33946-57.
14 Dang L, White DW, Gross S, et al. Cancer-associated IDH1 mutations produce 2-hydroxyglutarate. Nature 2009;462:739-44.

15 Molenaar RJ, Radivoyevitch T, Maciejewski JP, et al. The driver and passenger effects of isocitrate dehydrogenase 1 and 2 mutations in oncogenesis and survival prolongation. Biochim Biophys Acta 2014;1846:326-41.

16 Lee SM, Koh H-J, Park D-C, et al. Cytosolic NADP(+)-dependent isocitrate dehydrogenase status modulates oxidative damage to cells. Free Radic Biol Med 2002:32:1185-96.

17 Ronnebaum SM, Ilkayeva O, Burgess SC, et al. A pyruvate cycling pathway involving cytosolic NADP-dependent isocitrate dehydrogenase regulates glucose-stimulated insulin secretion. J Biol Chem 2006;281:30593-602.

18 Metallo CM, Gameiro PA, Bell EL, et al. Reductive glutamine metabolism by IDH1 mediates lipogenesis under hypoxia. Nature 2012;481:380-4.

19 Liu X, Ling Z-Q. Role of isocitrate dehydrogenase 1/2 (IDH 1/2) gene mutations in human tumors. Histol Histopathol 2015:30:1155--60.

20 Parsons DW, Jones S, Zhang $X$, et al. An integrated genomic analysis of human glioblastoma multiforme. Science 2008;321:1807-12.

21 Bleeker FE, Lamba S, Leenstra S, et al. IDH1 mutations at residue p.R132 (IDH1(R132)) occur frequently in high-grade gliomas but not in other solid tumors. Hum Mutat 2009;30:7-11

22 Ward PS, Patel J, Wise DR, et al. The common feature of leukemia-associated IDH1 and IDH2 mutations is a neomorphic enzyme activity converting alpha-ketoglutarate to 2-hydroxyglutarate. Cancer Cell 2010;17:225-34.

23 Lu C, Ward PS, Kapoor GS, et al. IDH mutation impairs histone demethylation and results in a block to cell differentiation. Nature 2012;483:474-8.

24 Koivunen P, Lee S, Duncan CG, et al. Transformation by the (R)-enantiomer of 2-hydroxyglutarate linked to EGLN activation. Nature 2012;483:484-8.

25 Turcan S, Rohle D, Goenka A, et al. IDH1 mutation is sufficient to establish the glioma hypermethylator phenotype. Nature 2012;483:479-83.

26 Xu W, Yang H, Liu Y, et al. Oncometabolite 2-hydroxyglutarate is a competitive inhibitor of $\alpha$-ketoglutarate-dependent dioxygenases. Cancer Cell 2011;19:17-30.

27 Balss J, Meyer J, Mueller W, et al. Analysis of the IDH1 codon 132 mutation in brain tumors. Acta Neuropathol 2008;116:597-602.

28 Brennan CW, Verhaak RGW, McKenna A, et al. The somatic genomic landscape of glioblastoma. Cell 2013;155:462-77.

29 Louis D, Ohgaki H, Wiestler $\mathrm{O}$, et al. WHO classification of central nervous system tumours. IARC: Lyon:, 2016.

30 Capper D, Zentgraf H, Balss J, et al. Monoclonal antibody specific for IDH1 R132H mutation. Acta Neuropathol 2009;118:599-601.

31 Camelo-Piragua S, Jansen M, Ganguly A, et al. A sensitive and specific diagnostic panel to distinguish diffuse astrocytoma from astrocytosis: chromosome 7 gain with mutant isocitrate dehydrogenase 1 and p53. J Neuropathol Exp Neurol 2011:70:110-5.

32 Capper D, Sahm F, Hartmann C, et al. Application of mutant IDH1 antibody to differentiate diffuse glioma from nonneoplastic central nervous system lesions and therapy-induced changes. Am J Surg Pathol 2010;34:1199-204.

33 Capper D, Weissert S, Balss J, et al. Characterization of R132H mutation-specific IDH1 antibody binding in brain tumors. Brain Pathol 2010;20:245-54.

34 Lee S. Diffuse gliomas for nonneuropathologists. Arch Pathol Lab Med 2018;142:804-14

35 Szuhai $\mathrm{K}$, Jennes I, de Jong $\mathrm{D}$, et al. Tiling resolution array-CGH shows that somatic mosaic deletion of the EXT gene is causative in EXT gene mutation negative multiple osteochondromas patients. Hum Mutat 2011;32:E2036-49.

36 Kerr DA, Lopez HU, Deshpande V, et al. Molecular distinction of chondrosarcoma from chondroblastic osteosarcoma through IDH1/2 mutations. Am J Surg Pathol 2013:37:787-95

37 Pellegrini AE, Drake RD, Qualman SJ. Spindle cell hemangioendothelioma: a neoplasm associated with Maffucci's syndrome. J Cutan Pathol 1995;22:173-6.

38 Amary MF, Damato S, Halai D, et al. Ollier disease and Maffucci syndrome are caused by somatic mosaic mutations of IDH1 and IDH2. Nat Genet 2011;43:1262-5.

39 Pansuriya TC, van Eijk R, d'Adamo P, et al. Somatic mosaic IDH1 and IDH2 mutations are associated with Enchondroma and spindle cell hemangioma in Ollier disease and Maffucci syndrome. Nat Genet 2011;43:1256-61.

40 Perkins P, Weiss SW. Spindle cell hemangioendothelioma. An analysis of 78 cases with reassessment of its pathogenesis and biologic behavior. Am J Surg Pathol 1996:20:1196-204.

41 Weiss SW, Enzinger FM. Spindle cell hemangioendothelioma. A low-grade angiosarcoma resembling a cavernous hemangioma and Kaposi's sarcoma. Am J Surg Pathol 1986;10:521-30.

42 Kurek KC, Pansuriya TC, van Ruler MAJH, et al. R132C IDH1 mutations are found in spindle cell hemangiomas and not in other vascular tumors or malformations. Am J Pathol 2013;182:1494-500.

43 Döhner H, Weisdorf DJ, Bloomfield CD. Acute myeloid leukemia. N Engl J Med 2015;373:1136-52

44 Zhang Y, Wei H, Tang K, et al. Mutation analysis of isocitrate dehydrogenase in acute lymphoblastic leukemia. Genet Test Mol Biomarkers 2012;16:991-5.

45 Graubert T, Walter MJ. Genetics of myelodysplastic syndromes: new insights. Hematology Am Soc Hematol Educ Program 2011;2011:543-9. 
46 Pardanani A, Lasho TL, Finke CM, et al. IDH1 and IDH2 mutation analysis in chronicand blast-phase myeloproliferative neoplasms. Leukemia 2010;24:1146-51.

47 Medeiros BC, Fathi AT, DiNardo CD, et al. Isocitrate dehydrogenase mutations in myeloid malignancies. Leukemia 2017;31:272-81.

48 Feng J-H, Guo X-P, Chen Y-Y, et al. Prognostic significance of IDH1 mutations in acute myeloid leukemia: a meta-analysis. Am J Blood Res 2012;2:254-64.

49 Thol F, Weissinger EM, Krauter J, et al. IDH1 mutations in patients with myelodysplastic syndromes are associated with an unfavorable prognosis. Haematologica 2010;95:1668-74.

50 Blechacz B, Gores GJ. Cholangiocarcinoma: advances in pathogenesis, diagnosis, and treatment. Hepatology 2008;48:308-21.

51 Voss JS, Holtegaard LM, Kerr SE, et al. Molecular profiling of cholangiocarcinoma shows potential for targeted therapy treatment decisions. Hum Pathol 2013;44:1216-22.

52 Mauzo SH, Lee M, Petros J, et al. Immunohistochemical demonstration of isocitrate dehydrogenase 1 (IDH1) mutation in a small subset of prostatic carcinomas. Appl Immunohistochem Mol Morphol 2014;22:284-7.

53 Toth LN, de Abreu FB, Tafe LJ. Non-small cell lung cancers with isocitrate dehydrogenase 1 or 2 (IDH1/2) mutations. Hum Pathol 2018;78:138-43.

54 Murugan AK, Bojdani E, Xing M. Identification and functional characterization of isocitrate dehydrogenase 1 (IDH1) mutations in thyroid cancer. Biochem Biophys Res Commun 2010;393:555-9.
55 Shibata T, Kokubu A. Mutant IDH1 confers an in vivo growth in a melanoma cell line with BRAF mutation. AJPA 2020;178:1395-402.

56 Dang L, Su S-SM, Su S. Isocitrate Dehydrogenase Mutation and (R)-2Hydroxyglutarate: From Basic Discovery to Therapeutics Development. Annu Rev Biochem 2017;86:305-31.

57 Boddu P, Borthakur G. Therapeutic targeting of isocitrate dehydrogenase mutant AML. Expert Opin Investig Drugs 2017;26:525-30.

58 Fan B, Mellinghoff IK, Wen PY, et al. Clinical pharmacokinetics and pharmacodynamics of ivosidenib, an oral, targeted inhibitor of mutant IDH1, in patients with advanced solid tumors. Invest New Drugs 2020;38:433-44.

59 Nicolay B, Narayanaswamy R, Amatangelo MD, et al. EXTH-34. combined use of the pan IDH mutant inhibitor AG-881 with radiation therapy shows added benefit in an orthotopic IDH1 mutant glioma model in vivo. Neuro Oncol 2017; 19:vi79.

60 Pusch S, Krausert S, Fischer V, et al. Pan-mutant IDH1 inhibitor BAY 1436032 for effective treatment of IDH1 mutant astrocytoma in vivo. Acta Neuropathol 2017;133:629-44.

61 Natsume A, Wakabayashi T, Miyakita Y, et al. Phase I study of a brain penetrant mutant IDH1 inhibitor DS-1001b in patients with recurrent or progressive IDHI mutant gliomas. J Clin Oncol 2019;37:2004. 